languages. A few of these will be described.

In addition to the usual data forms (including single- or double-precision integers and floating point), 8TRAN provides for bit strings of fixed length, character strings of variable or fixed length, and pointers. Pointer operations often permit programs to be considerably smaller than they would be otherwise and allow the possibility of list processing.

Input and output may be formatted but need not be. Default formats exist for output, and free form may be used for input.

Character manipulation and evaluation, including string extraction and comparison, are done as easily as are arithmetic operations. Comparison, concatenation, and Boolean operators are available, as are the other operators normally found in FORTRAN. Mixed-mode expressions may be used freely.

These features alone provide great flexibility in programming and therefore in experimental design. Others have a similar effect in terms of data storage. 8TRAN permits the user to define INPUT, OUTPUT, or UPDATE files, the latter being used for both input and output. The files may be addressed either in stream (ASCII characters) or in record (internal format) form. Thus, it is as easy to write, say, from core to disk as it is to receive input from a Teletype.

8TRAN makes very efficient use of core storage, something especially desirable with a small computer. Thus, the linking loader puts together only those subroutines that are required by the program. Temporary buffer storage is allocated dynamically during the execution of a program.

Although this description of 8TRAN is far from complete, it should provide some indication of its power and potential usefulness. Experience in our laboratory has already shown that when 8TRAN is employed, program-development time is a fraction of what it would be otherwise. Also, 8TRAN is considerably easier to learn than is Assembly language, especially if one has any familiarity with FORTRAN or PL/1. RATSS is currently being revised and generalized so that it may be one of the several monitors that can support the data-management concepts of 8TRAN." Thus, we feel that the range of empirical applications of the PDP-8 has been greatly enhanced.

\section{REFERENCES}

JOHNSON, E. S. Objective identification of strategy on a selection concept learning

task. Journal of Experimental Psychology, 1971, 90, 167-196.

JONES, L. V., JOHNSON, E. S., \&
YOUNG, F. W. Computer control of psychological experiments. Chapel Hill, N.C.: Psychometric Laboratory Report No. 80, November 1969.

RAPOPORT, A., JONES, L. V., \& KAHAN, J. P. Gambling behavior in multiple-choice multistage betting games. Journal of Mathematical Psychology, $1970,7,12-36$.

UTTAL, W. R. Misuse, abuse, overuse, and unuse of on-line computer facilities by psychologists. Behavior Research Methods \& Instrumentation, 1972, 4, 55-60.
WALLSTEN, T. S. Probabilistic information processing, Bayes' rule, and conjoint-measurement. Chapel Hill, N.C.: Psychometric Laboratory Report No. 98, August 1971.

YOUNG, F. W., \& CLIFF, N, Interactive scaling with individual subjects. Psychometrika, 1972, in press.

\section{NOTE}

1. It is anticipated that the revision will be completed by the time the conference proceedings are published.

\title{
GASPS: A General ASynchronous Processing System for the PDP-8*
}

\author{
THOMAS D. WICKENS \\ University of California, Los Angeles, California 90024 \\ and \\ JAMES HOWARD, GLENN RICE, and RICHARD B. MILLWARD \\ Brown University, Providence, Rhode Island 02912
}

Programs for the on-line control of human verbal-learning experiments are characterized by an abundance of short program segments interspersed with variable delays. Most operations (presentation of a stimulus, receipt of a response, even generation of feedback) require no more than a hundred machine instructions, but the sequence in which these basic sections are executed is usually more complicated and often cannot be determined in advance. This uncertain ordering of events is even more evident when several Ss are run simultaneously, but asynchronously, at their own pace. It is impossible to write such programs in linear languages such as FORTRAN, and the contingencies are usually sufficiently complex as to make the state-transition languages for operant experiments unworkable. What is needed is a way to control a sequence of contingent events, i.e., a program that allows relatively sophisticated scheduling of small program segments. Our attempt to develop such a system for the PDP-8 computer has resulted in the program reported here, GASPS, a General ASynchronous Processing System.

The original GASPS program was

*Please send requests for reprints or information to Professor Richard $B$. Millward, Department of Psychology, Brown University, Providence, Rhode Island 02912. A manual describing the use of GASPS in detail, with an example and a PAL listing of the program, is available from Professor Millward. Please send $\$ 2.50$ to cover duplication and mailing costs. A paper tape of the GASPS program (in PAL form) can be obtained for the same price. written to control four subject stations (keyboard, random-access slide projector, and indicator lights) from a PDP-8/S computer with a $4 \mathrm{~K}$ memory and an ASR-33 Teletype at the Human Learning Laboratory at Brown University. The only hardware addition to the computer other than the subject stations was a 12-bit clock that could be set by the program and would interrupt on overflow. Subsequently, the system has been extended to include a video display for each S, a high-speed paper-tape reader and punch, a $64 \mathrm{~K}$ random-access disk, and a magnetic-tape drive. Corresponding modifications have been made to GASPS, and the entire program now requires about 1,800 locations, leaving the remainder for the user's program. As noted below, the disk can be used to store program segments.

In a GASPS program, the short sections of code mentioned above are written as autonomous units, usually performing single functions. They will be referred to as tasks. Examples of tasks involved in simple learning experiments are selection of a stimulus, presentation of the stimulus, enabling the system to receive valid responses (after the stimulus has appeared), recording the S's response, presentation of feedback, generation of a record of the trial, and so forth. Control is passed to the tasks by the GASPS monitor and is returned to GASPS when the task is complete. When no task is pending execution, GASPS retains control.

Calls can also be made from a task to a number of subroutine entry points within GASPS. Three of these 
requests allow the task to schedule other tasks for execution, one allows first-in, first-out queues to be set up, and most of the remainder are concerned with input or output. This last class of routines services interrupts and queues output, freeing the user from the need to worry about such commonly used peripheral devices as the Teletype, disk, magnetic tape, and video displays. These routines are relatively straightforward and will not be discussed further.

The control of all external devices is handled through the use of the interrupt system, never through flag-checking loops. The interrupt is turned on during most of the time when GASPS is in control. Program tasks may be executed with the interrupt either on or off, depending on the importance of the task and its length. When an interrupt takes place, control is passed to a service routine. Many of these routines are within GASPS itself; the remainder are user-written tasks.

A task may be executed at any of several priority levels. The priority of a task is specified when it is scheduled and determines when it will be executed. There are four levels of priority, with Priority Level 0 tasks executed first and Priority Level 3 tasks last. Within a priority level, the oldest requests are serviced first. The usage of the priority levels is as follows: Level 0 -These tasks service interrupts from external devices and take control after time delays. They are never interruptable. Level 0 tasks should be short, generally doing no more than a few simple $I / O$ requests and scheduling a new task. Level 1-Tasks requested at this level are executed immediately following the Level 0 tasks. Level 1 tasks are usually noninterruptable and should be used for short control operations. Level 2-These are used for longer and less urgent tasks, usually run with the interrupt on. Level 3-These are the lowest priority tasks and would be used for the largest tasks. With a small system, such as the $4 \mathrm{~K}$ PDP- 8 , there appears to be little use for this priority level. When a task is interrupted, processing will be resumed only after the interrupt and any higher priority tasks have been serviced.

An important part of the GASPS system is the timer. GASPS maintains a record of the time since its initialization, making use, in part, of the external hardware clock. A task can obtain this time and can also request that another task be executed after a given time interval. At the end of the interval, control is passed to the new task. Because these tasks service timer interrupts, they have Priority Level 0.
The basic GASPS system limits the user to approximately $2 \mathrm{~K}$ of program space. This is sufficient for quite complicated programs but, in some cases, can be limiting. The current version of GASPS allows tasks to be resident on the disk as well as in core. When scheduled, these tasks are brought into unused pages in core and executed at Priority Level 2 . The page system of addressing in the PDP-8 means that single-page tasks are page-wise relocatable, so that the eventual location of disk-resident tasks in core is not important. For this reason, these tasks are limited to one page (128 instructions) in length.

At this time, tasks for use with GASPS must be coded in PDP-8 Assembly language (PAL). This makes the programming somewhat slower than with a higher-level language, although, since GASPS tasks are short single-purpose routines, they are usually easy to code. The logic of the program is usually coutained in the interrelation of the tasks, not in their content, and it is possible to develop a library of tasks that can be combined with little modification to form new programs.

\title{
The PLANIT computer-assisted instructional language: A potential tool of research
} in psychology

\author{
M. A. RAHIMI and T. A. FINO \\ Department of Computer Science, Michigan State University \\ East Lansing, Michigan $\mathbf{4 8 8 2 2}$
}

The purpose of this presentation is to acquaint psychologists with an existing programming language which is easily obtainable and which may be used for collecting data and administering tests to human Ss in a great number of situations.

The programming language to be discussed is PLANIT (Programming Language for Interactive Teaching), which was developed by the System Development Corporation under contract from the National Science Foundation. This system was recently installed at Michigan State University, and it is expected that it will be installed in more than a dozen universities and research centers by June.
The language is designed for writing programs for presentation of textual information to students and the reading and recording of their responses. The logic used by an author in his program can make use of the student's response, together with information stored on his past performance, to determine the next step in presentation of instructional material.

A program written in PLANIT is a set of instructions for directing the interaction between man and machine at a computer terminal. The recording of the interaction is done automatically. The PLANIT program can be punched on cards and loaded into the computer, or it can be

Example 1. Interactive preparation of a PLANIT program.

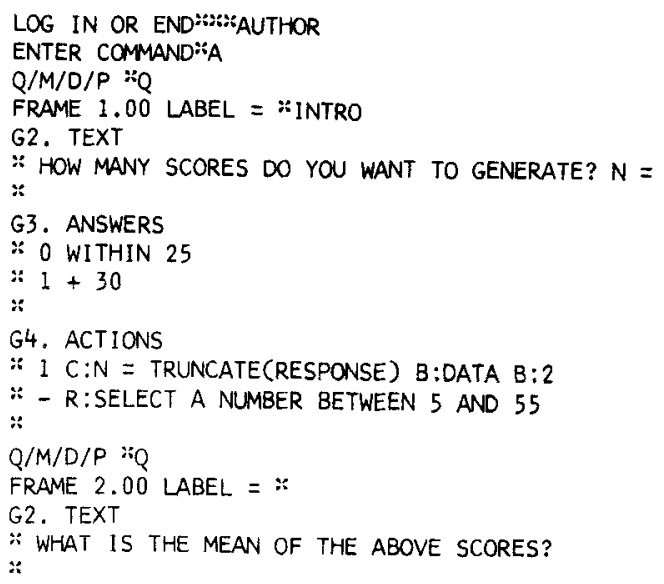

